\title{
An Approach to Calculate the Efficiency for an N- Receiver Wireless Power Transfer System
}

\author{
Thabat Thabet \\ School of Computer science and Electronic Engineering \\ University of Essex, Colchester, Uk \\ Technical Collage, Mosul, Iraq
}

\author{
Dr. John Woods \\ School of Computer science and Electronic Engineering \\ University of Essex \\ Colchester, Uk
}

\begin{abstract}
A wireless power transfer system with more than one receiver is a realistic proposition for charging multiple devices such as phones and a tablets. Therefore, it is necessary to consider systems with single transmitters and multiple receivers in terms of efficiency. Current offerings only consider single device charging systems. A problem encountered is the efficiency of one receiver can be affected by another because of the mutual inductance between them. In this paper, an efficiency calculation method is presented for a wireless power transfer system with one to $\mathbf{N}$-receivers. The mutual inductance between coils is implicitly calculated for different spatial positions and verified by practical experimentation. The effect of changing parameters, such as resonant frequency, coil size and distance between coils, on the efficiency has been studied. A clarification of the special performance of a wireless power transfer system at a specific point has been presented.
\end{abstract}

\section{Keywords - wireless power transfer; multiple receivers}

\section{INTRODUCTION}

Wireless power transfer by magnetic resonant frequency coupling is a new version of the classic theory presented by [1]. Wireless charging is more convenient and has a number of advantages over wired connections. In wireless charging there is no physical connection between the load and the source encouraging its use for no spark applications. Wireless power transfer depends on the near field and is convenient, safe, and simple [2]. Due to its dependence on magnetic resonance, Witricity is also safe from a biological point of view i.e. environmentally friendly [3]. The seminal work of this technique was conducted by Nikola Tesla in the late 19th century, he was one of the wireless power transmission pioneers [1].

In 2007 scientists at Massachusetts Institute of Technology (MIT) revisited the concept of wireless power transfer by announcing a testbed which wirelessly transferred tens of watts over a distance of greater than two meters. Following this, research in this area has received increased attention by many large multinational companies such as Apple, Duracell and Qualcomm [4]. Researchers have tended to study wireless power transfer systems with a single transmitter and a single receiver. It is clear that the performance of wireless power transfer changes according to factors such as the distance between the transmitter and the receiver [5-7].
In [8] the authors explained how one can express inductive coupling or magnetic resonance with the same analytic form as used in coupled mode theory. However, according to [9] this method is undesirable and inconvenient. Therefore researchers tend to use the equivalent circuit for a magnetic coupled system. For example, in [5] the researchers analysed an equivalent circuit wireless system and found its efficiency at different distances between the transmitter and the receiver demonstrating optimum performance at a specific point. In their analysis, s-parameters have been employed because they are more convenient for practical experiments at high frequency. In spite of the wealth of research to study efficiency for single receiver wireless systems, there is still a shortage of similar studies about multiple receivers.

In this paper, the equivalent circuit of the magnetic coupling system is proposed with some modification to study the efficiency of wireless power transfer with multiple receivers. An essential part of this study is the calculation of the mutual inductance between coils. Resonant frequency, coil size and distance between coils, and the effect of these parameters on efficiency has been considered. The optimum performance of the wireless power transfer system has been demonstrated for various cases.

The findings of our study can be used to form the basis of any multiple receiver system. The proposed method can also be applied analytically at different frequencies. To investigate the results practically, experiments have been conducted inside a Faraday cage at frequencies of $2.07 \mathrm{MHz}$ and $2.5 \mathrm{MHz}$. Choosing these frequencies is due to practical considerations such as a.c. signal sources and practical dimensions of the coils.

\section{WIRELESS POWER TRANSMISSION USING MAGNETIC RESONANCE}

The principle of the mutual induction phenomena, which happens between two coils, is the passing of a continuous time varying current through one of the coils called the primary coil leading to the production of a magnetic field around it. The interaction of this magnetic field with the second coil called secondary, leads to an induced current in it as shown in Fig.1. The maximum interaction happens when the two coils work at the same frequency and the mutual induction here is called magnetic resonance $[3,10]$. 


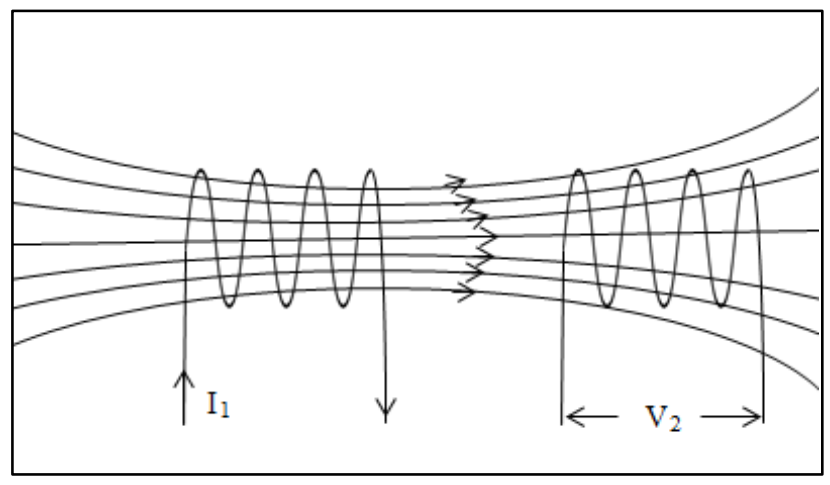

Fig. 1. Mutual Induction Process

\section{RESONANCE COUPLED SYSTEM REPRESENTATION}

\section{A. One load system representation}

A simple wireless power transmission system consists of a transmitter and a receiver as shown in Fig.2. The transmitter is represented by the source $V s$ and its internal resistor $R s$, the capacitor $C_{1}$ and the inductor $L_{1}$. While $C_{2}$ and $L_{2}$ are the capacitor and the inductor that achieve the resonance to transfer the power to the load resistor $R_{2}$.

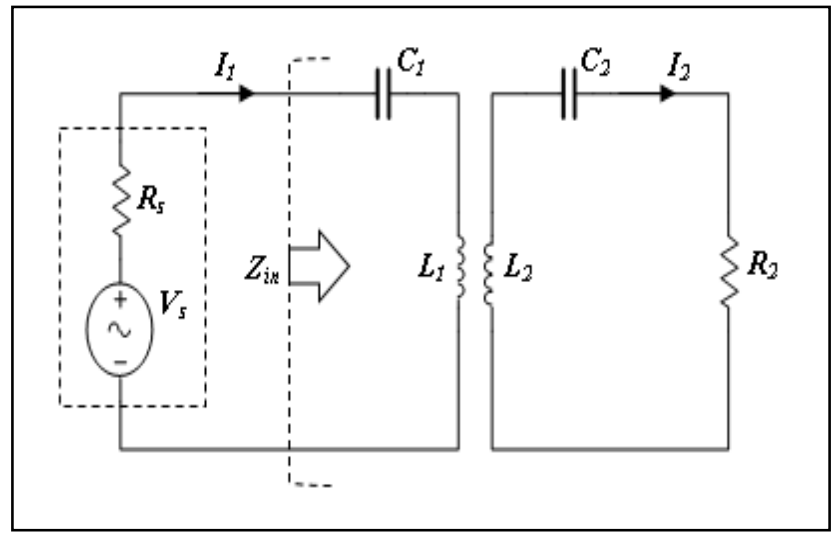

Fig. 2. Equivalent Circuit of a Wireless Power Transfer System

Due to the inductance value of each coil, capacitor values are chosen to achieve the resonance condition $X_{L}=X_{C}$, through the resonance frequency equation [11]:

$f_{o}=\frac{1}{2 \pi \sqrt{L_{1} C_{1}}}=\frac{1}{2 \pi \sqrt{L_{2} C_{2}}}$

\section{B. Two load system representation}

The same principle can be used to represent a system with two receivers or more. Fig. 3 shows a schematic circuit of a resonant coupled system which consists of a transmitter and two receivers. It shows the basic variables which are used in the calculation of mutual inductance. The variables $A$ and $a$ are the radius of the transmitter and each receiver, respectively. The distance between the transmitter and each receiver or between the two receivers are represented by $b$ and $D$, respectively. Finally, $p$ is the pitch between any two turns in any of the coils. It is clear from this circuit that the calculation method of mutual inductance between the transmitter and each receiver is different for the two receiver case due to variation in location. Different methods are proposed to calculate the mutual inductance for each case [12, $13]$.

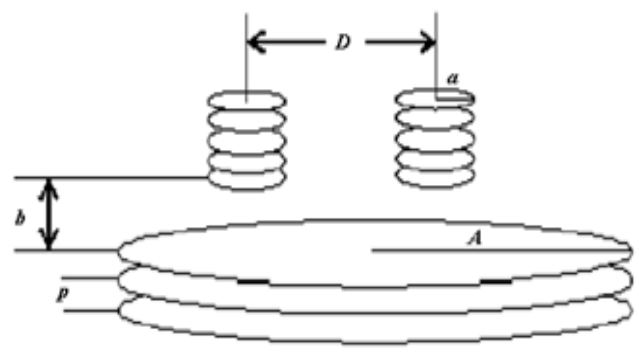

Fig. 3. Schematic Circuit of a Resonant coupled system

The equivalent circuit of a wireless power transfer system with two receivers is shown in Fig.4. In the figure, the source $V_{s}$ drives a transmitter coil and its internal resistor is $R_{s} . L_{l}$ and $C_{l}$ are the inductor and the capacitor of the transmitter. On the other side of the circuit, the first receiver is represented by $L_{2}$, $C_{2}$, and $R_{2}$ as an inductor, capacitor and load resistor, respectively. Similarly, $L_{3}, C_{3}$, and $R_{3}$ form the second receiver.

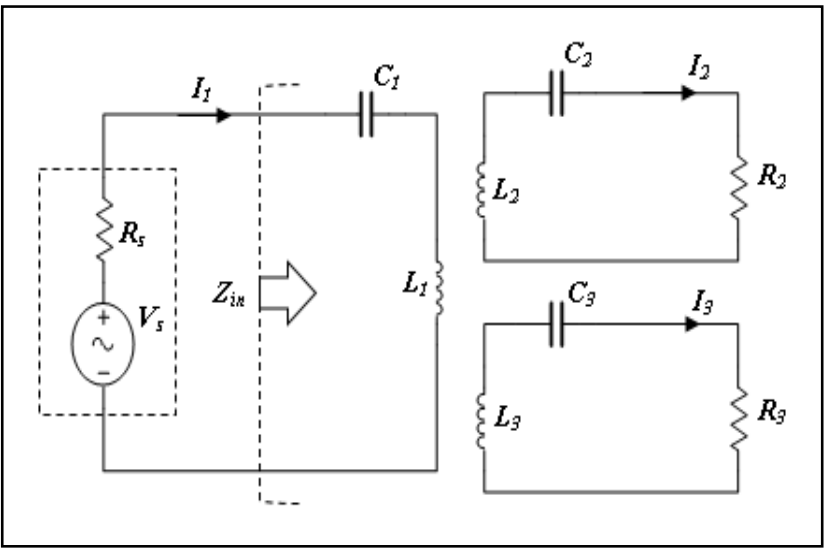

Fig. 4. Equivalent Circuit of a Wireless Power Transfer System with Two Receivers

The second receiver should be under the resonance condition with the same resonance frequency.

$f_{o}=\frac{1}{2 \pi \sqrt{L_{3} C_{3}}}$

\section{BASICS OF THEORETICAL ANALYSIS}

Applying Kirchhoff's law of voltage on each loop [14] (the transmitter and the receiver) is the start of the analysis. $M_{12}$ or $M_{21}$ is the mutual inductance between the transmitter and the receiver.

$$
\begin{aligned}
& V_{s}=\left(R_{s}+j w L_{1}+\frac{1}{j w C_{1}}\right) I_{1}-j w M_{12} I_{2} \\
& 0=-j w M_{21} I_{1}+\left(R_{2}+j w L_{2}+\frac{1}{j w C_{2}}\right) I_{2}
\end{aligned}
$$

Where $\mathrm{w}=2 \pi \mathrm{f}$ 
$I_{1}$ and $I_{2}$ : The loops currents.

Applying Z-parameters on this set of equations leads to:

$\left[\begin{array}{c}V_{s} \\ 0\end{array}\right]=\left[\begin{array}{ll}Z_{11} & Z_{12} \\ Z_{21} & Z_{22}\end{array}\right]\left[\begin{array}{l}I_{1} \\ I_{2}\end{array}\right]=[Z]\left[\begin{array}{l}I_{1} \\ I_{2}\end{array}\right]$

The previous equation can be written as:

$\left[\begin{array}{l}I_{1} \\ I_{2}\end{array}\right]_{\text {So }}=\operatorname{inv}[Z]\left[\begin{array}{c}V_{s} \\ 0\end{array}\right]$

$I_{1}=\operatorname{inv} Z(1,1) V_{S}$

$I_{2}=\operatorname{inv} Z(2,1) V_{s}$

It is concluded from (6-a) that:

$Z_{\text {in }}=\operatorname{inv} Z(1,1)-R_{S}$

Here, the output and input power can be calculated:

$P_{\text {out }}=I_{2}^{2} R_{2}$

$P_{\text {in }}=\left(\frac{V_{S}}{R_{S}+Z_{\text {in }}}\right)^{2} Z_{\text {in }}$

Under the condition of maximum transfer of power, the efficiency can be calculated as:

eff $=\operatorname{inv} Z(2,1)^{2}\left(R_{S}+R_{2}\right)^{2} * 100 \%$

\section{TheORETICAL ANAlysis of MUltiPle ReCEIVERS}

Following the same theoretical analysis as for a single receiver system we extend the method for a number of the receivers; this is the main contribution of this paper.

\section{A. Two Receivers}

For the circuit shown in Fig.3, Kirchhoff's law of voltage is applied on each loop (the transmitter and the two receivers) with regard to the mutual inductance between each pair of coils [14]. The mutual inductance between the transmitter and the first, second receiver are $M_{12}$ and $M_{13}$, respectively. Both of them follow the same calculation method because of their relation to the transmitter. As a special case, $M_{12}$ is equal to $M_{13}$ if the two receivers are identical. While $M_{23}$, the mutual inductance between the two receivers has to be calculated using another method due to their parallel nature.

$V_{s}=\left(R_{s}+j w L_{1}+\frac{1}{j w C_{1}}\right) I_{1}-j w M_{12} I_{2}-j w M_{13} I_{3}(11-\mathrm{a})$

$0=-j w M_{21} I_{1}+\left(R_{2}+j w L_{2}+\frac{1}{j w C_{2}}\right) I_{2}+j w M_{23} I_{3}$

$0=-j w M_{31} I_{1}+j w M_{32} I_{2}+\left(R_{3}+j w L_{3}+\frac{1}{j w C_{3}}\right) I_{3} \quad(11-\mathrm{c})$

Where $w=2 \pi f$

$I_{1}, I_{2}$ and $I_{3}$ : The loops currents.

Applying Z-parameters on this set of equations leads to:

$\left[\begin{array}{c}V_{s} \\ 0 \\ 0\end{array}\right]=\left[\begin{array}{lll}Z_{11} & Z_{12} & Z_{13} \\ Z_{21} & Z_{22} & Z_{23} \\ Z_{31} & Z_{32} & Z_{33}\end{array}\right]\left[\begin{array}{l}I_{1} \\ I_{2} \\ I_{3}\end{array}\right]=[Z]\left[\begin{array}{l}I_{1} \\ I_{2} \\ I_{3}\end{array}\right]$

The previous equation can be written as:
$\left[\begin{array}{l}I_{1} \\ I_{2} \\ I_{3}\end{array}\right]=\operatorname{inv}[Z]\left[\begin{array}{l}V_{s} \\ 0 \\ 0\end{array}\right]$

So

$I_{1}=\operatorname{inv} Z(1,1) V_{S}$

$I_{2}=\operatorname{inv} Z(2,1) V_{S}$

$I_{3}=\operatorname{inv} Z(3,1) V_{s}$

It is concluded from (14-a) that:

$Z_{\text {in }}=\operatorname{inv} Z(1,1)-R_{S}$

Here, the output power for each receiver can be calculated:

$P_{\text {out } 1}=I_{2}^{2} R_{2}$

$P_{\text {out } 2}=I_{3}^{2} R_{3}$

And the input power is:

$P_{\text {in }}=\left(\frac{V_{s}}{R_{S}+Z_{\text {in }}}\right)^{2} Z_{\text {in }}$

Under the condition of maximum transfer of power, the efficiency for each receiver can be calculated as:

eff $f_{1}=\operatorname{inv} Z(2,1)^{2}\left(R_{S}+R_{2}\right)^{2} * 100 \%$

eff $f_{2}=\operatorname{inv} Z(3,1)^{2}\left(R_{s}+R_{3}\right)^{2} * 100 \%$

\section{B. Three Receivers}

Similar representation for the two receivers system can be applied on the three receivers system:

$\begin{aligned} V_{S}= & \left(R_{S}+j w L_{1}+\frac{1}{j w C_{1}}\right) I_{1}-j w M_{12} I_{2}-j w M_{13} I_{3}-j w M_{14} I_{4} \\ 0= & -j w M_{21} I_{1}+\left(R_{2}+j w L_{2}+\frac{1}{j w C_{2}}\right) I_{2}+j w M_{23} I_{3}+j w M_{24} I_{4} \\ 0= & -j w M_{31} I_{1}+j w M_{32} I_{2}+\left(R_{3}+j w L_{3}+\frac{1}{j w C_{3}}\right) I_{3}+j w M_{34} I_{4} \\ 0= & -j w M_{41} I_{1}+j w M_{42} I_{2}+j w M_{43} I_{3}+\left(R_{4}+j w L_{4}+\frac{1}{j w C_{4}}\right) I_{4} \\ & \text { Where } \mathrm{w}=2 \pi \mathrm{f}\end{aligned}$

$I_{1}, I_{2}$ and $I_{3}:$ The loops currents.

Applying Z-parameters on this set of equations leads to:

$\left[\begin{array}{l}V_{s} \\ 0 \\ 0 \\ 0\end{array}\right]=\left[\begin{array}{llll}Z_{11} & Z_{12} & Z_{13} & Z_{14} \\ Z_{21} & Z_{22} & Z_{23} & Z_{24} \\ Z_{31} & Z_{32} & Z_{33} & Z_{34} \\ Z_{41} & Z_{42} & Z_{43} & Z_{44}\end{array}\right]\left[\begin{array}{c}I_{1} \\ I_{2} \\ I_{3} \\ I_{4}\end{array}\right]=[Z]\left[\begin{array}{c}I_{1} \\ I_{2} \\ I_{3} \\ I_{4}\end{array}\right]$

The previous equation can be written as:

$\left[\begin{array}{c}I_{1} \\ I_{2} \\ I_{3} \\ I_{4}\end{array}\right]=\operatorname{inv}[Z]\left[\begin{array}{l}V_{s} \\ 0 \\ 0 \\ 0\end{array}\right]$

$I_{1}=\operatorname{inv} Z(1,1) V_{S}$

$I_{2}=\operatorname{inv} Z(2,1) V_{s}$

$I_{3}=\operatorname{inv} Z(3,1) V_{s}$

$I_{4}=\operatorname{inv} Z(4,1) V_{s}$

It is concluded from (22-a) that:

$Z_{\text {in }}=\operatorname{inv} Z(1,1)-R_{s}$ 
Here, the output power for each receiver can be calculated:

$$
\begin{aligned}
& P_{\text {out } 1}=I_{2}^{2} R_{2} \\
& P_{\text {out } 2}=I_{3}^{2} R_{3} \\
& P_{\text {out } 3}=I_{4}^{2} R_{4}
\end{aligned}
$$

And the input power is:

$P_{\text {in }}=\left(\frac{V_{S}}{R_{S}+Z_{\text {in }}}\right)^{2} Z_{\text {in }}$

Under the condition of maximum transfer of power, the efficiency for each receiver can be calculated as:

eff $f_{1}=\operatorname{inv} Z(2,1)^{2}\left(R_{S}+R_{2}\right)^{2} * 100 \%$

eff $f_{2}=\operatorname{inv} Z(3,1)^{2}\left(R_{s}+R_{3}\right)^{2} * 100 \%$

eff $f_{3}=\operatorname{inv} Z(2,1)^{2}\left(R_{S}+R_{2}\right)^{2} * 100 \%$

\section{C. $N$-Receivers}

The method can be applied in the general case for an $\mathrm{N}$ receiver system and the general equation for any system is:

$$
\left[\begin{array}{c}
V_{s} \\
0 \\
0 \\
\vdots \\
0
\end{array}\right]=\left[\begin{array}{ccccc}
Z_{11} & Z_{12} & Z_{13} & \ldots & Z_{1 n} \\
Z_{21} & Z_{22} & Z_{23} & \ldots & Z_{2 n} \\
Z_{31} & Z_{32} & Z_{33} & \ldots & Z_{3 n} \\
\vdots & \vdots & \vdots & \ldots & \vdots \\
Z_{n 1} & Z_{n 2} & Z_{n 3} & \ldots & Z_{n n}
\end{array}\right]\left[\begin{array}{c}
I_{1} \\
I_{2} \\
I_{3} \\
\vdots \\
I_{n}
\end{array}\right]
$$

\section{Mutual Inductance Methods}

\section{A. Concentrical Coils}

According to [5], The Neumunn formula can be applied to find the mutual inductance between two concentric turns:

$$
\begin{aligned}
& M=\frac{\mu_{o}}{4 \pi} \iint \frac{d l_{1} d l_{2}}{D} \\
& \quad \text { Where } \mu_{o}=4 \pi * 10^{-7} \mathrm{H} / \mathrm{m} \\
& D \text { : the distance between } d l_{1} \text { and } d l_{2}
\end{aligned}
$$

The same solution for this integral can be achieved by the Maxwell equation [12]:

$$
M_{12}=\mu_{o} \sqrt{A a}\left(\frac{2}{k}-k\right) K(k)-\frac{2}{k} E(k)
$$

Where $A, a$ : the radius of what is regarded as two concentric turns as shown in Fig.2

$$
k=\frac{2 \sqrt{A a}}{\sqrt{(A+a)^{2}+D^{2}}}
$$

$K$ and $E$ : the first and second kind of the complete elliptic integral and they are defined as:

$$
\begin{gathered}
K=\int_{0}^{\pi / 2} \frac{d \varphi}{\sqrt{1-k^{2} \sin ^{2} \varphi}} \\
E=\int_{0}^{\pi / 2} \sqrt{1-k^{2} \sin ^{2} \varphi} d \varphi
\end{gathered}
$$

As a modification to deal with a coil of more than one turn, the following expression was used instead of $D$ in (30):

$$
\begin{aligned}
& D \rightarrow D+(j-1) p+(i-1) p \\
& \text { Where } i=1 \rightarrow N_{1} \\
& \quad j=1 \rightarrow N_{2} \\
& N_{l} \text { and } N_{2} \text { : the number of turns for each coil. } \\
& P: \text { the pitch between turns }
\end{aligned}
$$

Finally, the total mutual inductance between two concentric coils with multiple turns can be found by [12]:

$M_{\text {total }}=\sum_{i=1}^{N_{1}} \sum_{j=1}^{N_{2}} M_{i j}$

\section{B. Parallel Coils}

Mutual inductance calculation between two parallel coils needs another formula instead of the Maxwell equation. In [13], the researchers presented two formulas to express the mutual inductance between two turns at the same level and two turns with lateral misalignment.

1) Two turns at the same level

The following formula can be applied to calculate the mutual inductance between two turns at the same level:

$$
\begin{array}{r}
M=\frac{\mu_{o} a_{p}}{2 \pi} \int_{0}^{\pi} \sqrt{(1-\alpha V)^{2}+\beta^{2}}\left[\left(1-\frac{k^{2}}{2}\right) K(k)-E(k)\right] d \varphi \\
\text { Where } \\
\alpha=\frac{a_{s}}{a_{p}} \quad \beta=\frac{C}{a_{p}} \\
k^{2}=\frac{4 \alpha V}{(1+\alpha V)^{2}+\beta^{2}} \\
V=\sqrt{1+\frac{d^{2}}{a_{s}^{2}}-\frac{2 d}{a_{s}} \cos \varphi}
\end{array}
$$

In this case $C$ equals zero because the two turns are in the same level

\section{2) Lateral Misalignment}

If the two turns are allocated at different levels, the mutual inductance between them can be calculated by:

$M=\frac{\mu_{o}}{\pi} \sqrt{a_{p} a_{s}} \int_{0}^{\pi} \frac{\left(1-\frac{d}{a_{s}} \cos \varphi\right)}{\sqrt{V^{3}}}\left(\frac{2}{k}-k\right) K(k)-\frac{2}{k} E(k) d(34)$

Again the mutual inductance between each of the two turns of the two parallel coils can be summed by (32) to find the total mutual inductance.

\section{RESULTS AND DISCUSSION}

The representation of a wireless power transfer system with one to n-receivers is programmed in Matlab to calculate the mutual inductance between coils and then the efficiency.

\section{A. Single Receiver System}

As a starting point to investigate the previous analysis, a wireless power transfer system with two identical coils is practically implemented. Each coil, with $3 \mathrm{~cm}$ radius, 21 turns and $3 \mathrm{~mm}$ pitch between the turns, has an inductance equal to $18.6 \mu \mathrm{H}$. One of them is the transmitter and the other is the receiver. Choosing specific parameters such as resonance 
frequency at $2.07 \mathrm{MHz}$, load resistor $50 \Omega, C_{1}=C_{2}=330 \mathrm{pF}$ (which includes the natural capacitance of the coil) and the distance between the two coils is $3 \mathrm{~cm}$. Next, finding the efficiency over a range of frequencies was achieved as shown in Fig.5. The theoretical results are compared with the experimental in the figure.

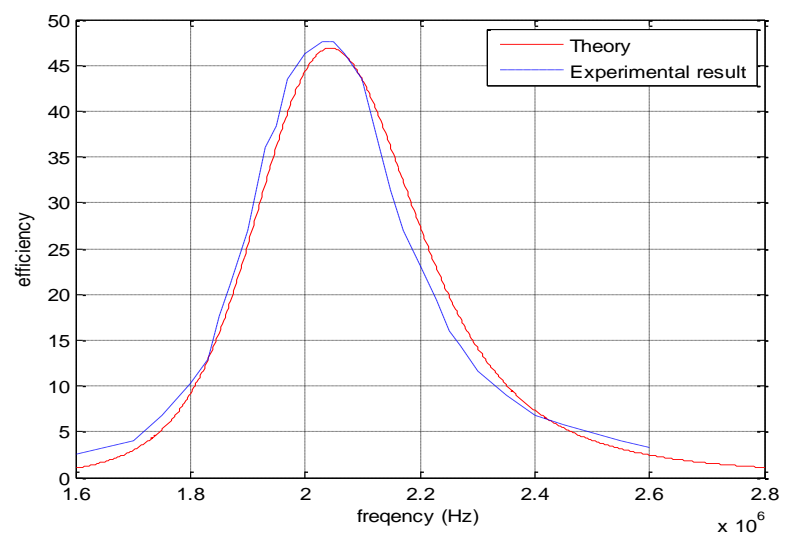

Fig. 5. Efficiency versus frequency at $3 \mathrm{~cm}$ distance and $\mathrm{f}_{\mathrm{o}}=2.07 \mathrm{MHz}$

It is clear from Fig. 5 that the higher efficiency of $46 \%$ for wireless transfer happens at the resonance frequency and there is a good match between the theoretical and practical results. However, the efficiency here is relatively low, that is because of the small value of mutual inductance between the two coils. It is equal to $1.5468 \mu \mathrm{H}$.

Replacing the two coils with another set of coils, which have higher inductance and higher mutual inductance between them, gives higher efficiency over larger distances at the same frequency as shown in Fig.6. In this experiment, each coil has $12.25 \mathrm{~cm}$ radius, 8 turns and $3 \mathrm{~mm}$ pitch between the turns, and $31.3 \mu \mathrm{H}$ inductance. Working at the same frequency of $2.07 \mathrm{MHz}, 188 \mathrm{pF}$ capacitors are required for the transmitter and receiver. The system has an efficiency of $72 \%$ at $15.5 \mathrm{~cm}$. This is as a result of the $2.1583 \mu \mathrm{H}$ mutual inductance, which is larger compared to the previous experiment.

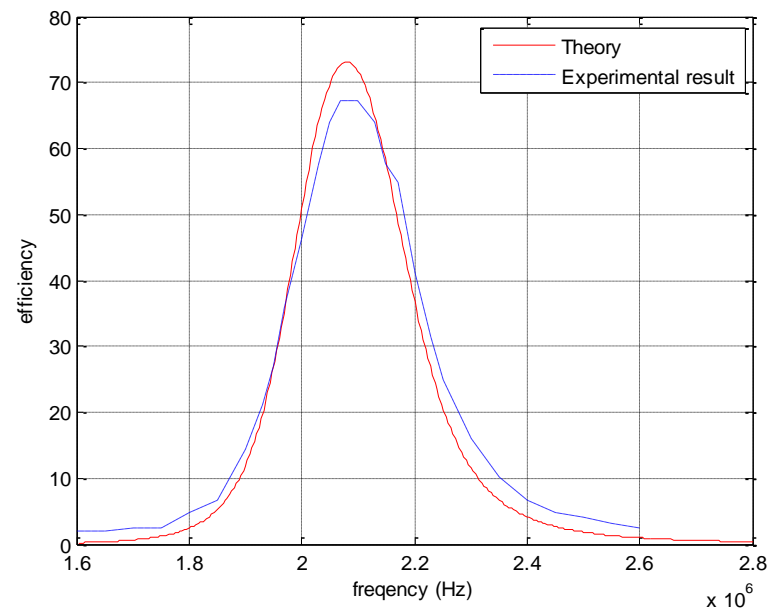

Fig. 6. Efficiency versus frequency at $15.5 \mathrm{~cm}$ distance and $\mathrm{f}_{\mathrm{o}}=2.07 \mathrm{MHz}$ (Mutual Inductance Effect)
It was explained in section 6 that mutual inductance increases when the two coils approach. Fig.7 shows the effect of changing the distance between these two coils on the mutual inductance.

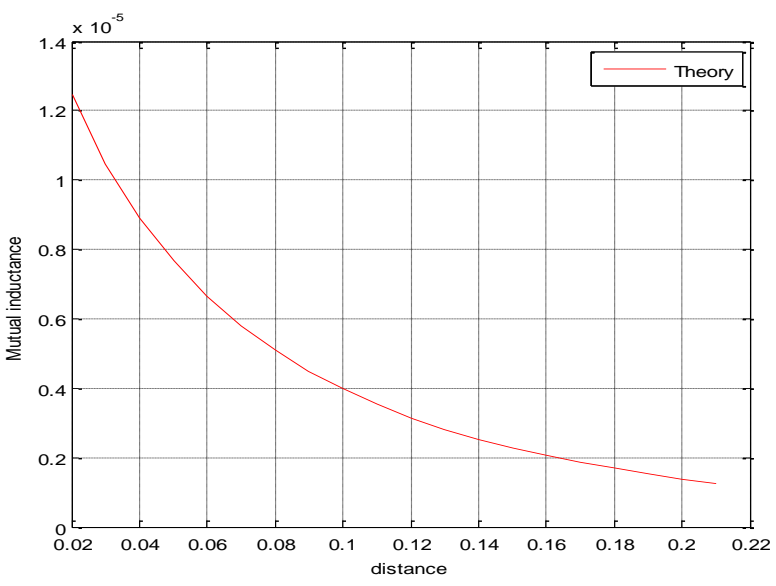

Fig. 7. Mutual inductance versus distance

Using the larger coils as in Fig.6, at smaller distances, the efficiency at the same resonant frequency gradually increases until it approaches the maximum value of $100 \%$ at a distance of $10 \mathrm{~cm}$ as shown in Fig. 8 .

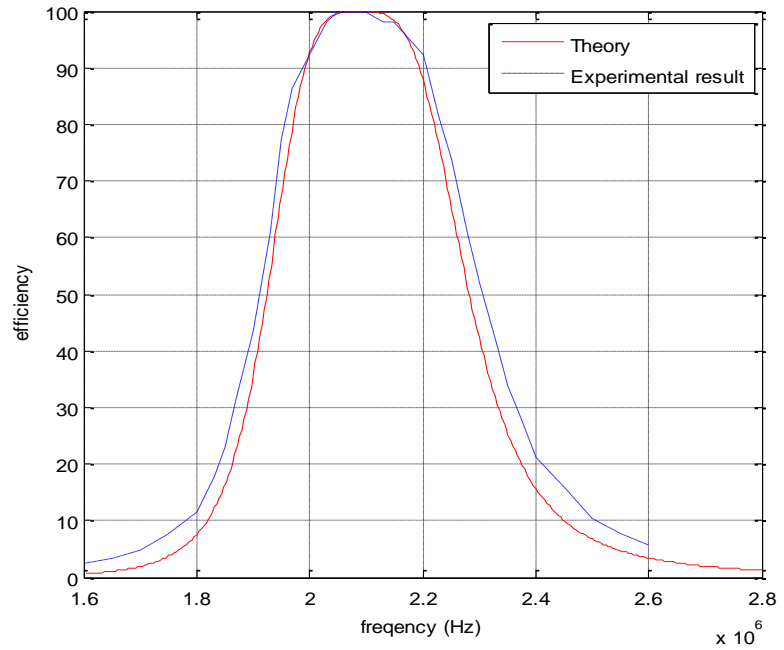

Fig. 8. Efficiency versus frequency at $10 \mathrm{~cm}$ distance and $\mathrm{f}_{\mathrm{o}}=2.07 \mathrm{MHz}$ (Distance Effect)

A further decrease in the distance surprisingly leads to a decrease in efficiency of the system. As shown in Fig.9, there is a splitting in the efficiency of the system at a distance of $3.5 \mathrm{~cm}$. This splitting starts to appear as the mutual inductance increases between the two coils (the transmitter and the receiver), increasing when the two coils are approaching. This effect shifts the maximum efficiency to two distinct frequencies around the resonance frequency. This means that there is a perfect point to transfer the maximum power in the wireless system as shown in Fig.10 from the relation between the efficiency and the distance at the resonant frequency. 


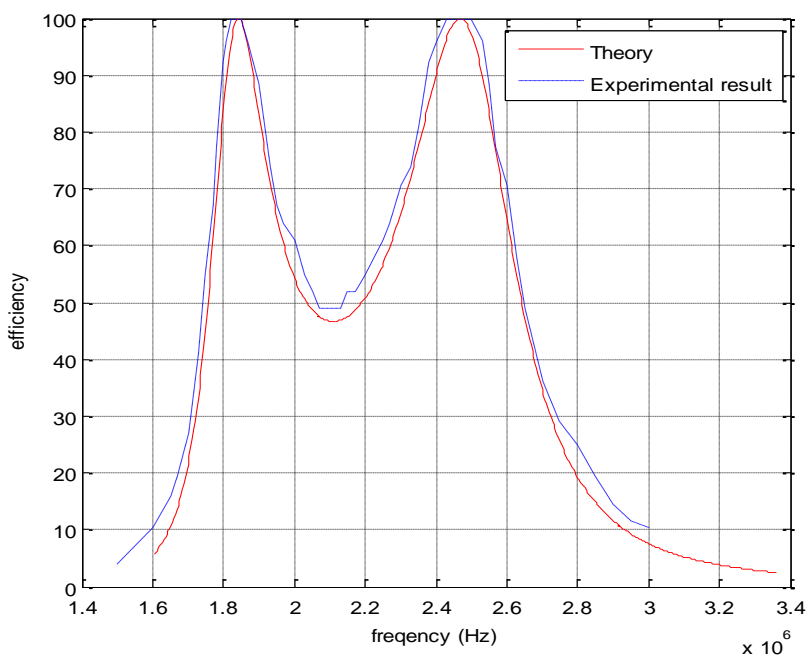

Fig. 9. Efficiency versus frequency at $3.5 \mathrm{~cm}$ distance and $\mathrm{f}_{\mathrm{o}}=2.07 \mathrm{MHz}$

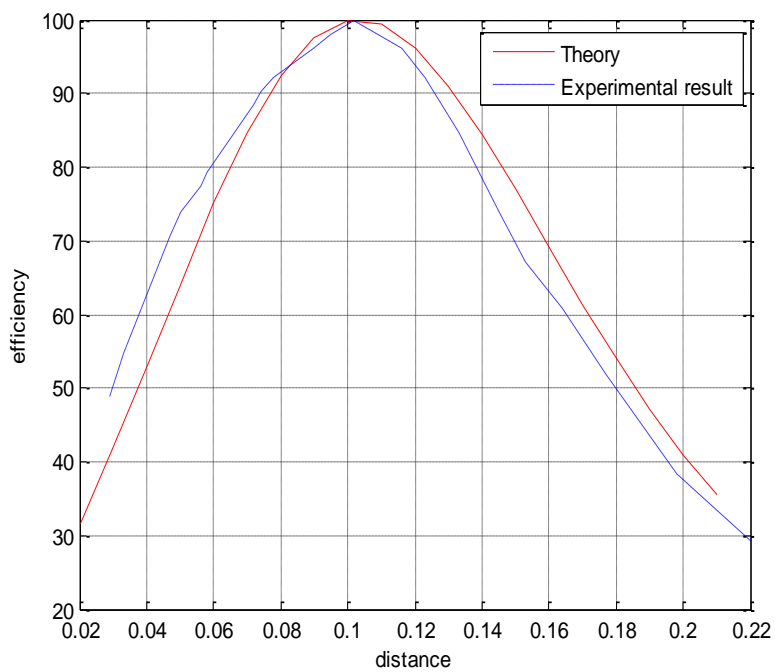

Fig. 10. Efficiency versus distance at resonant frequency

The reason for this point is shown in Fig.11, where the input resistance of the system, which changes with the distance, equals $50 \Omega$ at this point. The $50 \Omega$ value is equal to the input resistance of the source $\mathrm{Z}_{\text {in }}$ which leads to maximum transfer of power.

Working at a different frequency will also affect the efficiency of the wireless power transfer system. To show this effect, the capacitors of the transmitter and the receiver are changed from $188 \mathrm{pF}$ to $128 \mathrm{pF}$. The results are shown in Fig.12. The efficiency of the system is shown at the resonant frequency of $2.5 \mathrm{MHz}$ with $15.5 \mathrm{~cm}$ distance between coils. Compared to Fig. 6 and Fig.8, the higher frequency enables the system to transfer more power through a larger distance. It can be concluded that the distance of the perfect point increases at higher frequency but is ultimately limited by the frequency and mutual inductance in both coils.

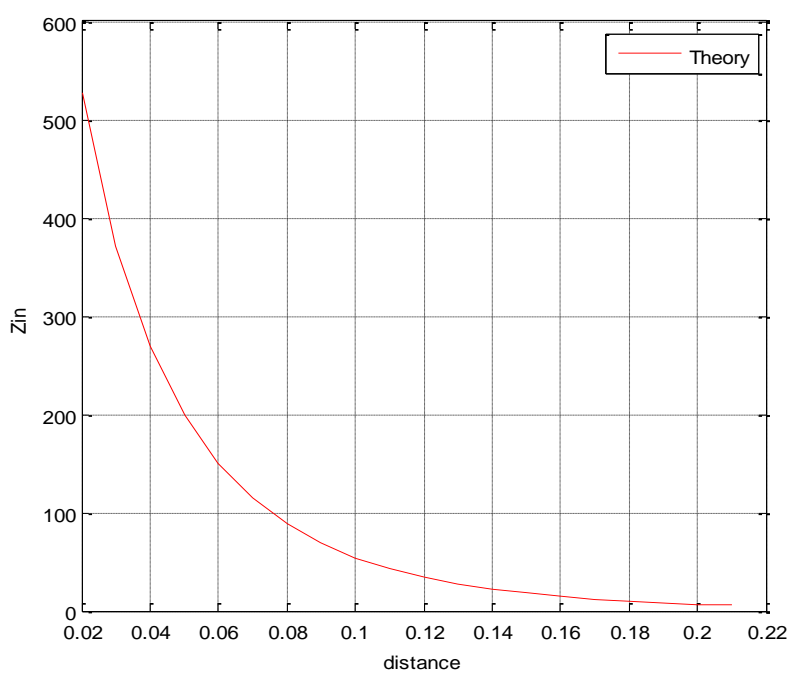

Fig. 11. Input resistance versus distance at resonant frequency

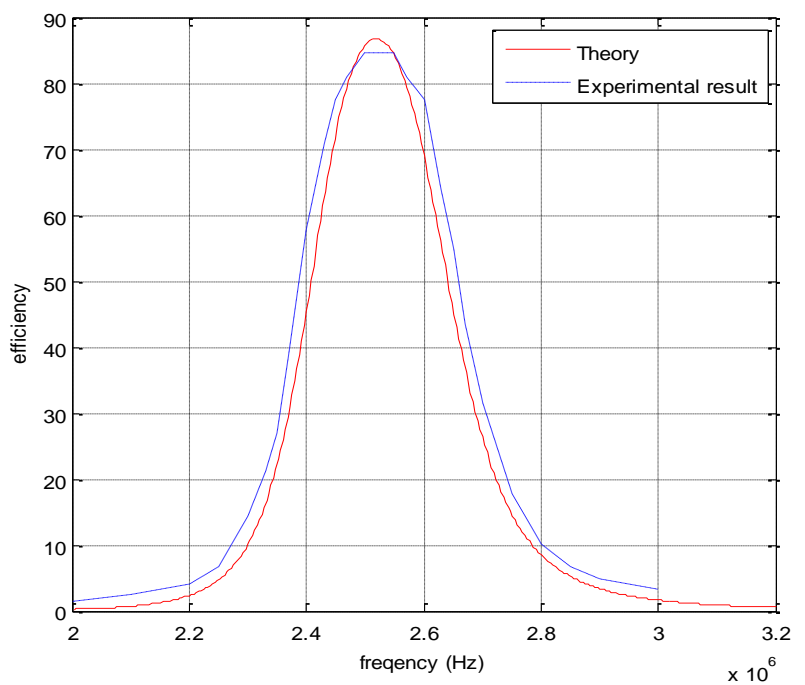

Fig. 12. Efficiency versus frequency at $15.5 \mathrm{~cm}$ distance and $\mathrm{f}_{\mathrm{o}}=2.5 \mathrm{MHz}$ (Resonance Frequency Effect)

Before studying multiple receivers it is necessary to study the efficiency of a small receiver with a large transmitter. The efficiency of a system with the large coil of $12.25 \mathrm{~cm}$ and the small coil of $3 \mathrm{~cm}$ is shown in Fig. 13 at a resonant frequency of $2.07 \mathrm{MHz}$ and a distance of $3 \mathrm{~cm}$.

\section{A. Two Receiver System}

Using two identical receivers of $3 \mathrm{~cm}$ radius and a transmitter of $12 \mathrm{~cm}$ the efficiency of a two coil system is studied. The efficiency of a receiver can be affected by the presence of another receiver in the same field. Fig.14 shows the efficiency of each one of two closed receivers in the same transmitter field. The distance between their centres is $7 \mathrm{~cm}$. It appears from the figure that the efficiency here is less than it is in case of just one receiver as shown in Fig.13. 


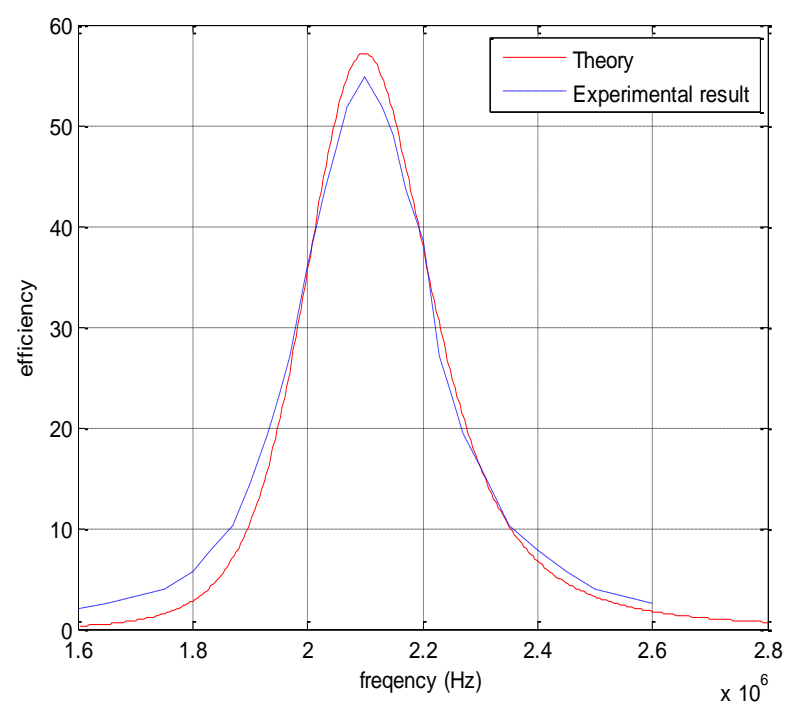

Fig. 13. Efficiency versus frequency at $3 \mathrm{~cm}$ distance
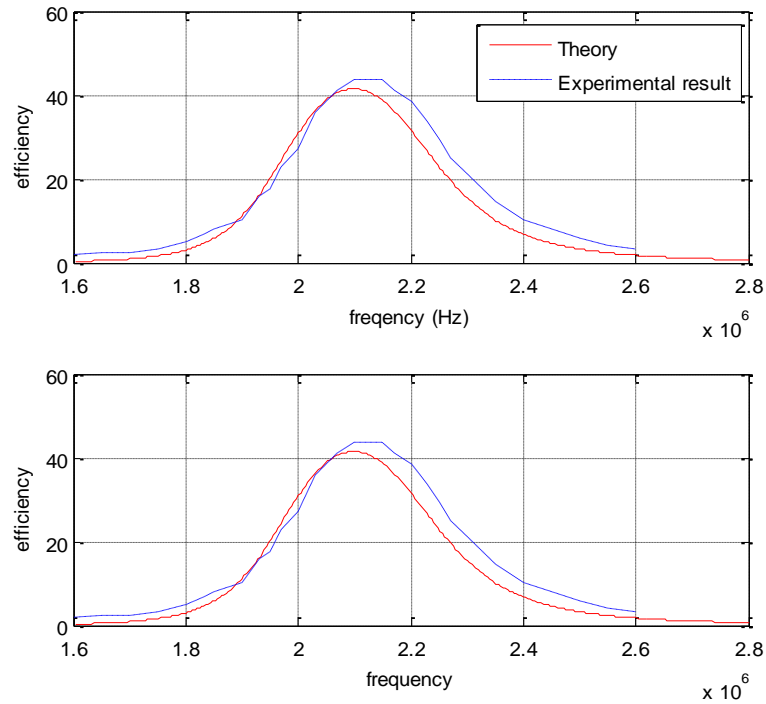

Fig. 14. Efficiency versus frequency for two receivers system

\section{B. Three Receiver System}

It is possible to apply our modelling and simulation approach for the case of more than two receivers, i.e. three to N. Fig. 15 shows the efficiency of one of the three receivers if all three are together inside the field of the same transmitter. In this part the three identical receivers form a triangular shape at the same level. This means that the mutual inductance between any two of them are equal, which is $0.21076 \mu \mathrm{H}$. Moreover, the mutual inductance between the transmitter and any one of the receivers are also equal which is $1.7358 \mu \mathrm{H}$. Moving a receiver to another location in the same field requires a new calculation for the mutual inductance between coils.
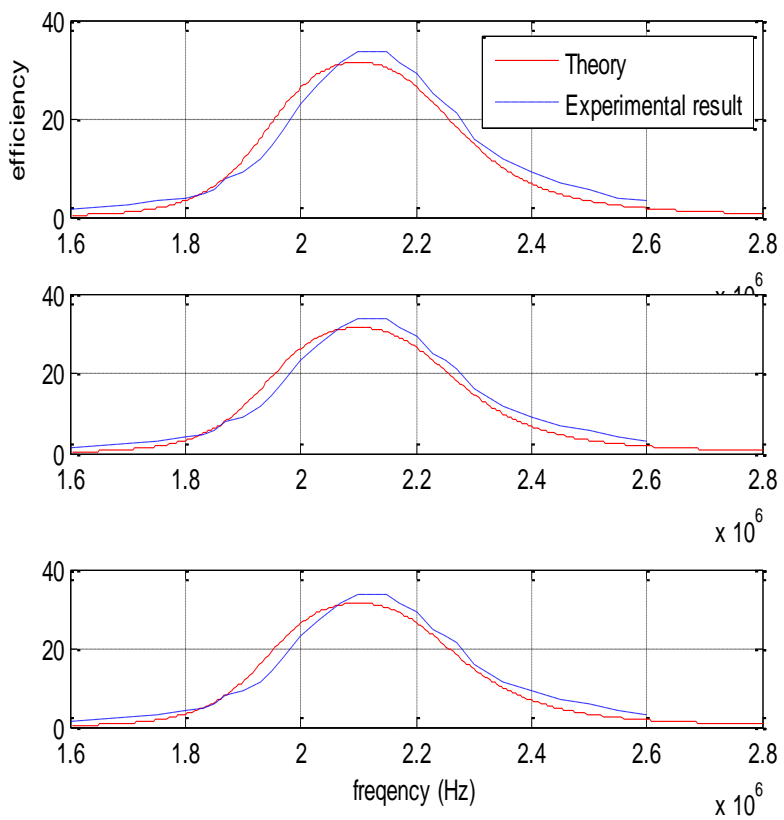

Fig. 15. Efficiency versus frequency for three receivers system

\section{CONCLUSION}

The mutual inductance between any two coils depends on their shape, their location and the distance between them. This study presented a method for the calculation of mutual inductance between any solenoid coils with a different location and distance.

The efficiency of a wireless power transfer system is dependent on factors such as: the resonance frequency; the size of the coils; the mutual inductance between them; the distance between coils and the presence of more than one receiver in the transmitter area. The following observations can be made:

- There is a specific distance, where the input resistance of the system equals the internal resistor of the source, at which point the system has the maximum efficiency and can transfer maximum power to the load.

- Higher frequency leads to an increase in the distance at which the system has the maximum efficiency subject to frequency and mutual inductance limitations.

- A Larger transmitter size, produces more flux around it, and also leads to an increase in the distance at which the system has the maximum efficiency.

- The Presence of more than one receiver gives decreased efficiency for each individual (compared to the larger coil), but the sum is greater than the efficiency of the same individual coil

- There are a number of practical limitations observed at higher frequencies regarding measurements 
Applying the proposed method is an efficient and compact way to calculate the efficiency for a wireless power transfer system with one to $\mathrm{N}$ - receivers.

\section{REFERENCES}

[1] T. W. Versloot, D. J. Barker, and X. O. One, "Optimization of Near-Field Wireless Power Transfer Using Evolutionary Strategies," The 8th European Conference on Antennas and Propagation, Netherlands, 2014.

[2] J. Wang, S. Ho, W. Fu, and M. Sun, "Analytical design study of a novel WiTricity charger with lateral and angular misalignments for efficient wireless energy transmission," Magnetics, IEEE Transactions on, vol. 47, no. 10, pp. 2616-2619, 2011.

[3] A. Mahmood, A. Ismail, Z. Zaman, H. Fakhar, Z. Najam, M. Hasan, and S. Ahmed, "A Comparative Study of Wireless Power Transmission Techniques," Journal of Basic and Applied Scientific Research, vol. 4, no. 1, pp. 321-326, 2014.

[4] O. Rönnbäck, "Optimization of Wireless Power," 2013.

[5] T. Imura, and Y. Hori, "Maximizing air gap and efficiency of magnetic resonant coupling for wireless power transfer using equivalent circuit and neumann formula," Industrial Electronics, IEEE Transactions on, vol. 58, no. 10, pp. 4746-4752, 2011.

[6] V. Jiwariyavej, T. Imura, and Y. Hori, "Coupling Coefficients Estimation of Wireless Power Transfer System via Magnetic Resonance Coupling using Information from Either Side of the System," in Proc. International Conference on Broadband and Biomedical Communication, Australia, 2012.
[7] D. Chaurasia, and S. Ahirwar, "An Optimal Parameter Estimation Technique for Wireless Electricity Transmission," Research India Publications, vol. 3, no. 1, pp. 1-9, 2013.

[8] H. Shim, J. Park, S. Nam, and B. Lee, “A criterion Proposed for Inductive Coupling and Magnetic Resonance Coupling in Wireless Power Transfer System, " Microwave Conference (APMC), 2014 Asia-Pacific. IEEE, 2014.

[9] T. Beh, M. Kato, T. Imura, and Y. Hori, "Wireless Power Transfer System via Magnetic Resonant Coupling at Fixed Resonance Frequency-Power Transfer System Based on Impedance Matching_," EVS-25 Shenzhen, China, 2010.

[10] J. Gozalvez, "WiTricity-the wireless power transfer [Mobile radio]," Vehicular Technology Magazine, IEEE, vol. 2, no. 2, pp. 38-44, 2007.

[11] M. Yasir, and M. S. Haque, "The Witricity: Revolution in Power Transmission Technology," International Journal of Scientific \& Engineering Research, vol. 4, no. 8, pp. 565-570, 2013.

[12] A. C. M. de Queiroz, "Mutual inductance and inductance calculations by Maxwell's Method," Home page of Dr. Antonio Carlos M. de Queiroz, 2005.

[13] C. Akyel, S. I. Babic, and M.-M. Mahmoudi, "Mutual inductance calculation for non-coaxial circular air coils with parallel axes," Progress In Electromagnetics Research, vol. 91, pp. 287-301, 2009.

[14] Y. Zhang, Z. Zhao, and K. Chen, "Frequency-Splitting Analysis of FourCoil Resonant Wireless Power Transfer," Industry Applications, IEEE Transactions on, vol. 50, no. 4, pp. 2436-2445, 2014. 